Elsevier required licence: (C) $<2020>$. This manuscript version is made available under the CC-BY-NCND 4.0 license http://creativecommons.org/licenses/by-nc-nd/4.0/

The definitive publisher version is available online at

[https://linkinghub.elsevier.com/retrieve/pii/S1871519218316561] 


\section{Working with women: semi-structured interviews with Australian complementary medicine maternity care practitioners}

\section{Abstract}

Problem: Despite the known prevalence of complementary medicine use by women during pregnancy and childbirth and the evolution of preferred models of maternity care, very little is known about the nature and characteristics of the care provided to women by complementary medicine practitioners during this important life stage.

Background: Woman-centred care is a speciation of person-centred care which has achieved prominence in maternity care policy in recent years. There is also evidence that the core principles of some complementary medicine systems of medicine emphasise patient-centredness and that these principles are core drivers toward complementary medicine use in multiple populations. Aim: This study aims to explore the approach to care delivered by complementary medicine practitioners to women during pregnancy and birth. Methods: Semi-structured individual interviews were conducted with 23 complementary medicine practitioners who identified as specialising in maternity care. Data from the interviews were analysed using a framework approach. Findings: The analysis of the perspective of complementary medicine practitioner's experiences providing care to pregnant and birthing women identified three main themes: Responding to women's expectations of care; providing woman-centred care; and the therapeutic relationship at the heart of woman-centred care. Discussion: The approach to maternity care reported by complementary medicine practitioners aligns with the principles of woman-centred care, possibly due to the similarities between woman-centred care and the core features of many systems of medicine within complementary medicine. Conclusion: complementary medicine practitioners may contribute to an overall experience of woman-centred maternity care for pregnant and birthing women.

Keywords: complementary therapies, maternity care, patient-centred, qualitative, woman-centred 
1. Introduction

Statement of Significance

\begin{tabular}{|l|l|}
\hline Problem or Issue & $\begin{array}{l}\text { Despite the known prevalence of complementary medicine } \\
\text { use by women during pregnancy and childbirth very little is } \\
\text { known about the care provided to women by complementary } \\
\text { medicine practitioners during this important life stage }\end{array}$ \\
\hline $\begin{array}{l}\text { What is already } \\
\text { known }\end{array}$ & $\begin{array}{l}\text { Woman-centred care focuses on: women's individual needs } \\
\text { and expectations; recognizes the need for women to have } \\
\text { choice, control and continuity of care; and recognises } \\
\text { women's expertise in decision-making. Complementary } \\
\text { medicine practice is suggested to align with the principles of } \\
\text { patient-centred care. }\end{array}$ \\
\hline $\begin{array}{l}\text { What this paper } \\
\text { adds }\end{array}$ & $\begin{array}{l}\text { Complementary medicine practitioners and the women who } \\
\text { consult with them for maternity care place importance on } \\
\text { woman-centred care. Complementary medicine practitioners } \\
\text { emphasise informed decision-making and values continuity of } \\
\text { care. }\end{array}$ \\
\hline
\end{tabular}

Woman-centred care is a speciation of person-centred care which has achieved prominence in maternity care policy in recent years $[1,2]$. Woman-centred care is a concept that focuses on the woman's individual needs and expectations, rather than the needs of institutions or professionals; recognizes the need for women to have a choice, control and continuity of care; and recognises the woman's expertise in decision-making [2]. In line with interest in woman-centred care among policy makers, there is growing evidence for the value and benefit of informed decisionmaking [3] and continuity of care, whereby care is provided by the same health professional throughout pregnancy and birth [4].

Complementary medicine $(\mathrm{CM})$ - a range of treatments and practices not commonly included in conventional medical training [5] - is used by a large proportion of women during pregnancy and birth [6], including significant levels of consultations with $\mathrm{CM}$ practitioners $[7,8]$. Given $\mathrm{CM}$ practitioners may be providing care to women 
for pregnancy-related health conditions during the antenatal, intrapartum and postnatal period, the role of $\mathrm{CM}$ practitioners as providers of maternity care - defined by the Australian Department of Health as care which includes antenatal, intrapartum and postpartum care for women and babies as delivered in a variety of public and private settings [9] - requires closer examination. Within the scope of these definitions, the types of $\mathrm{CM}$ practitioners providing maternity care may include not only those commonly associated with $\mathrm{CM}$ - naturopaths, massage therapists, acupuncturists - but also other maternity-specific practitioner groups that exist outside of traditional maternity care training such as doulas $[7,8]$. Some evidence suggests individuals may choose the services of a CM practitioner because of the alignment between CM practice and the principles of patient-centred care [10]. Other research indicates that the perception that $\mathrm{CM}$ has greater safety compared to conventional maternity care may influence women's decision to use CM [11, 12].

The exact nature and level of detail required by women to support informed decisionmaking may vary based on differences in health literacy whereby women with higher levels of health literacy may seek more information before making a decision [13, 14]. In parallel, individuals who choose to use CM are frequently reported to have higher levels of education and health literacy when compared with non-users [15]. There is also evidence that the core principles of some CM systems of medicine emphasise the education of patients and empowering active engagement with health behaviours [16-18], both of which are core requirements of informed decision-making [19], and that these principles are core drivers toward CM use in multiple populations.[20]

Despite the known prevalence of CM use by women during pregnancy and childbirth and the evolution of preferred models of maternity care, very little is known about the 
nature and characteristics of the care provided to women by CM practitioners during this important life stage.[21] As such, this study presents the perceptions and experiences of their approach to maternity care by a range of CM practitioners.

\section{Methods}

Aim

This study aims to explore the approach to care delivered by CM practitioners to women during pregnancy and birth.

\section{Recruitment}

Recruitment was conducted via relevant practitioner associations (e.g. Australian Traditional Medicine Society, Australian Natural Therapists Association, National Herbalists Association of Australia, Osteopathy Australia, Australian Association of Massage Therapists, Chiropractic Association of Australia), through an 'expression of interest' email. The associations were selected based on the alignment between their membership base and the sampling frame, as well as the number of members.

\section{Sample}

Twenty-three interested CM practitioners in current clinical practice in south-east Queensland responded to the recruitment drive, all of whom were included in the study (see Table 1).

\section{Data collection}

Semi-structured interviews were conducted by [redacted for blinded review] using an interview guide with $\mathrm{CM}$ practitioners who identified as specialising in maternity care. The female interviewer was a PhD candidate at the time of data collection. She was trained in qualitative research and had previous experience conducting semistructured interviews of health professionals. There was no prior relationship with the 
participants beyond the interaction involved in organising the interview except in rare circumstances where the participant had met the interviewer at a professional event. All interviewees were informed of the interviewer's background and qualifications as it related to this study as well as their motivations for undertaking the research project. The interview guide was developed to address the research questions of the wider project by [redacted for blinded review] with input from [redacted for blinded review] and a third experienced sociologist not included in the authorship of this paper. The guide was pilot tested with a CM practitioner known to the interviewer before data collection commenced with no changes to the guide required. The domains covered included identity as practitioner, interprofessional communication, and women in care. The time and location of interviews was chosen to suit the participants and varied between individuals. Interviews were recorded via a digital recorder and then transcribed. Each interview lasted between 45 and 60 minutes. It was found that thematic saturation was attained with 15 participants, however all interested practitioners were interviewed to ensure any differences between practitioner groups was captured.

\section{Data analysis}

Thematic data analysis was undertaken in line with the Framework approach [22] which is an established approach used in health services research including within maternity services research [23-25]. In line with this approach, the thematic analysis followed a process of familiarisation, identifying a thematic framework, indexing, charting, and mapping and interpretation [22]. [Redacted for blinded review] undertook immersion in the raw data by listening to recorded interviews and reading transcripts. The data were then imported into N-Vivo software, charted to identify themes and analysed for intersecting concepts. Finally, researcher theme 
triangulation was undertaken to ensure consistency of analysis, in which [redacted for blinded review] analysed a de-identified excerpt of interviews, and different interpretations were identified, discussed and consensus achieved. Quotes were selected based upon the quality of the quote and the representativeness of the theme.

\section{Ethical clearance}

Ethical clearance was granted by human research ethics committees from [redacted for blinded review], [redacted for blinded review] and [redacted for blinded review].

\section{Results}

The analysis of the perspective of $23 \mathrm{CM}$ practitioner's experiences providing care to pregnant and birthing women identified three main themes: Responding to women's expectations of care; providing woman-centred care; and the therapeutic relationship at the heart of woman-centred care.

\subsection{Responding to women's expectations of care}

Women were perceived by participants to have varying expectations of care from their health professionals in general, and their CM practitioner in particular. Participants described the challenge of meeting and responding to the women's expectations of the care provided by all health professionals. Women, it was argued, have high expectations of their maternity care provider as they are primarily paying clients in contrast to free or heavily subsidised provision of hospital-based and general practitioner (GP) care and this reflects in the client-practitioner relationship.

"I think [they have high expectations] especially if they are seeing an obstetrician and they're paying for it."-Naturopath2 
This dynamic between women as a paying client and their expectations of health professionals also extended across to the public system but was considered to create differing expectations of care depending on the degree of 'out-of-pocket' expenses women were outlaying. Ultimately, this was described as creating a different standard of care expected between health professions.

$$
\begin{aligned}
& \text { "Well they're probably not paying to see a GP or midwife } \\
& \text { anyway because that's through the public health system, } \\
& \text { because they're paying to see me, their expectation of my } \\
& \text { care is probably going to be quite high!"-Naturopath1 }
\end{aligned}
$$

Counter to this, a view was also proposed that differences in expectations were linked more to the women's perceptions of the potential value or approach of the CM practitioner rather than economic factors.

"I don't know if they...expect the same thing, because we don't offer the same thing. They might say, "I don't really care [who] the obstetrician [is], as long as they've got good clinical skills because at the end of the day, that's what I need from them," whereas from their midwife, or their naturopath, or whoever else that is providing more of an emotional supportive role, or you know, someone they can get on with and really click with, that's the niche as well, I suppose."-Naturopath2

\subsubsection{Managing women's expectations}

Participants also described the challenges associated with managing this expectation among pregnant women in their care. Clear information at the initiation of a therapeutic relationship was described by a number of participants as important 
for ensuring women have realistic expectations of care. Some practitioners emphasised establishing women's expectations and responding to them early as being a primary focus of their attempts to manage expectations:

"You also really clearly establish their expectations of you, and what you're actually going to be providing, because I think it's really important you make that clear upfront."-Doula10

Whilst other practitioners described clearly stating their own expectations of the care they are providing and ensuring women are agreeable to the proposed outcomes:

"And they'll normally come in for an initial consultation, which we'll do a history, an examination, and really find out from them what is their particular issue, and what they'd like help with. And then provide them with "ok this is what I think is going on, this is the way l'd like to approach it, does that sound ok with you? Have you got any concerns?" and "I would expect it would take this amount of treatments to improve your condition." -Chiropractor13

When women's expectations were not aligned with the outcomes practitioners felt they could provide, the importance of communicating this to the woman was highlighted by participants:

"I think some people are, they want to know black and white "when is it going to be better? Tell me exactly how many times I need to come," that type of thing. But I just say to them "I can't tell the future!" (laughs) 'You're an individual, l've never treated a 'you' before, I 
don't know how you're going to respond... we're just going to have to see how your body reacts."-Chiropractor21

\subsection{Providing woman-centred care}

The importance of providing care that emphasises and supports women's autonomy whilst also acknowledging women's existing values and perceptions was described by most participants. This focus on womancentred care is evident in the comments from this massage therapist and acupuncturist.

“I can offer suggestions, but I feel it's important that every pregnant woman be in some way feeling that she's yeah, driving the whole journey herself."-Massage6

"I find good maternity care is trying to figure out what she wants, and if you can provide it"-Acupuncture14

\subsubsection{Educating women and their families}

The most common characteristic of care which participants said they employed to best provide woman-centred care was educating women and their families. In some instances, this focused on providing women with adequate information to make informed decisions about their maternity care. This doula described her process of educating women and their partners about the options available to them as an key aspect of the care she provides:

"I totally support the family in what they want to do. I do offer them options and I'll ask them if they know there are options, because often people don't know."-Doula16 
The women who were perceived to be more actively involved in their maternity care were often described as prioritising informed-decision making and would expect quality information from their health provider. Where the information provided was considered inadequate these women were said to source additional information on their own until they felt comfortable, that they could make an informed decision. In other circumstances the practitioner prioritised training the woman and/or her partner in practical skills which could be employed at home or in the birth environment:

"I'll sometimes teach women moxa, that they can take home. Or I get their partners to come in, and I'll teach them, so that they can do treatment at home, if that's useful."-Acupuncturist15

This emphasis on education also extended to empowering women to affect health behaviours such as diet and lifestyle changes:

"Because if you explain it really well, as to why they need to do this and you keep on them about it, then usually you can get some sort of a change. Normally you can at least get part way, yeah."-Acupuncturist17

\subsubsection{Supporting balanced decision-making}

The challenge participants described, when providing information to women, is the need to do so in a manner which supported balanced decision-making rather than trying to sway women to a particular outcome. The importance of maintaining this separation between the clinician's personal views and the care provided to women was emphasised by this Doula: 
If they still choose something that I wouldn't choose or wouldn't recommend then that's up to them. They have employed me to support them in that and so I will."-Doula16

Although some participants also acknowledged that they still hold their own views and these may inadvertently be communicated to the woman:

"And I you know, I've got to be a little bit careful, because I have patients who come in and say "I'm having a caesarean," and I ask their reasons why and...make sure they have an understanding of their choices, without saying "you're wrong," because if that's their choice then that's their choice. I might not agree with it, but it's not my baby."-Osteopath7

At times, however, participants indicated a need to support a woman's empowerment, so she can engage with other maternity care providers to find the answers to her questions and access the care that is most appropriate to her own values and needs. For example, some participants described encouraging women to approach their conventional maternity care provider for clarification or further information about proposed treatments. An example of this approach was described by a chiropractor participating in the study:

"...then they do start asking questions, and then it's like you know, "well maybe you should talk about this with your obs [obstetrician] rather than [me]" but they don't feel like they can sometimes" - Chiropractor13

\subsection{The therapeutic relationship at the heart of woman-centred maternity care}

Underpinning the other two main themes was the importance of establishing and developing a positive therapeutic relationship between the practitioner and the pregnant woman. The therapeutic relationship was positioned as a key element in 
providing effective woman-centred maternity care. A driving factor in the successful establishment of the desired therapeutic relationship was practitioner-woman rapport because it supported the woman to disclose important health concerns and also allowed the practitioner to better identify how to support women in affecting changes to her health behaviours which benefit the pregnancy and the baby. This dynamic is described by a naturopath:

“Well its primary I think, because you can't have a therapeutic relationship with someone without rapport, and that means that they trust and respect your opinion and you know, you're supporting and managing them, and that's a team effort I suppose."-Naturopath1

The same practitioner then proceeded to describe the impact of rapport on disclosure of detrimental lifestyle behaviours:

"It [rapport] probably affects...the things that they tell me, they're probably very honest with me about you know, any bad habits that they might have" Naturopath1

The nature of the therapeutic relationship and how it develops is also approached in a 'woman-centred' manner by most practitioners as well, in that they respond to the needs of the woman and reshape the relationship in accordance with that. The resulting rapport was positioned by some practitioners as more profound than women experience with conventional maternity care providers:

"They will open up more probably to me than maybe their doctors, and sometimes to their friends you know, because there's just things they want to talk about."-Massage18 


\subsubsection{Establishing the therapeutic relationship}

As the quality of therapeutic relationship was considered a vital component to the care provided to women, participants also described the factors they perceived impacted on establishing a positive therapeutic relationship. Good listening skills and empathy were two such factors described by participants. This chiropractor describes the need to show understanding - or empathy - and compassion as integral to providing care to women:

"The biggest qualities that they [a maternity health professional] need to have, compassion; they need to be understanding; they need to be very careful. A lot of the stuff that they need to have is merely aiming to make sure that that mum and that child walk away healthy, healthier, and happy." -Chiropractor20

Alongside the practitioner interpersonal skills, the value of time was frequently acknowledged as an important enabler for the participants to develop the necessary rapport with the pregnant women in their care. A massage therapist participant describes the impact that the duration of the consultation has on women's sharing of information with their care provider:

"You're offering something that's just so personal, and they really do open up to you, probably...because of the time they spend with you."-Massage18

The value of these two factors - practitioner empathy and consultation time - were viewed as equally important and the absence of these were posited to be potentially undermining the quality of health care being delivered by conventional health professionals: 
"But I think most of the time, people don't listen to what people want, or fob them off. And it might just be a time thing, or it might just be that they're not a very empathetic practitioner."-Acupuncturist19

\subsubsection{The previous care relationship as a foundation for antenatal care}

This concept of time relates not only to the duration of consultations but also the temporal and ongoing nature of the therapeutic relationship and the continuing health benefits of lifestyle changes, often beginning in the preconception period and continuing through after birth as the family grows. This previous therapeutic relationship was seen as important foundation for the antenatal care relationship:

"Well in some respects, I see women before they are pregnant, so obviously during the pre-conception care, so that's where I would start the care I guess. And then other times, you would just see women at some point during the pregnancy for assistance with pregnancy complaints, or looking at birth support, or nutritional advice...then usually a postnatal check, and lactation support, if that's needed as well"-Naturopath2

In many cases, practitioners have been treating women previously for other health complaints, leading women to consult with them for pregnancy-related complaints as well:

"We have a long history, l've been treating her for quite a while, for various things, mainly anxiety and depression over the years."-Naturopath3

The reason that women continue to consult with a CM practitioner once pregnant following historical treatment for, in some cases, unrelated health complaints was linked by participants back to the established therapeutic relationship and the resulting trust women place in the practitioner: 
"I guess it makes it easier...because they trust me." -Naturopath23

In other cases, participants felt it was their pre-existing relationship which allowed them to explain the potential benefits of their care for addressing specific pregnancy-related health complaints were not well managed within conventional maternity care, such as this acupuncturist describing the process of explaining the effectiveness of acupuncture for the treatment of nausea and vomiting in pregnancy:

"Again, not a lot of people know about it, so if they're already patients, I'm trying to educate them early that acupuncture can be useful for that sort of stuff."-Acupuncturist15

\subsection{Safety is paramount}

When providing care to pregnant women alongside other maternity care professionals, participants described the need to be able to draw on evidence to clearly explain safety and risk to women, their families and other health professionals. Safety was described consistently as a vitally important consideration when providing care to pregnant women:

"Yeah, oh it's paramount, that you know safety is an absolute priority in pregnancy, or in treating anyone really, but during pregnancy obviously it's highly important." -Naturopath2

However, concerns about safety were not something that participants identified as raised by women among their client base, beyond initial general questions during their first consultation. As such, participants commonly identified that concerns for the woman's safety was directed by the practitioner more so than the woman: 
"Safety? Look rarely at all, but I usually bring that up in the beginning anyway. So I discuss things like bruising, bleeding that sort of stuff in the beginning...especially if it's someone's first acupuncture visit." Acupuncturist17

An exception to this was reported by an acupuncturist who reported that partners may drive questions about safety of $\mathrm{CM}$ treatments:

"It's usually their husbands. I have certainly, on many occasions had women ring and say, "oh I'm not going to continue with treatment, because my husband doesn't want me to risk damaging the baby," and that's just born from ignorance."-Acupuncturist15

\section{Discussion and Conclusion}

\subsection{Discussion}

This study presents a novel exploration of the experience and perspective of CM practitioners providing care to pregnant women. A key finding from the analysis is the importance placed on woman-centred care by CM professionals and women in their care. This model of care is linked to the larger movement within contemporary health systems and policy towards patient-centred care [26]. Support for patient-centred care, or woman-centred care in the case of maternity care, is evident among numerous leading health organisations globally and is reflected in a shift in policy and practice in maternity care towards a stronger focus on shared decision-making [1]. However, despite women's desire for more holistic maternity service delivery, barriers such as the increased medicalisation of births within facilities focused on organisational policies rather than women's need for respectful care [27] limit the degree to which this desire manifests within women's maternity care experience. The finding that CM practitioners reported an approach to care which aligns with a woman- 
centred model is unsurprising given the philosophical similarity between womancentred care principles and the core principles of many CM systems of medicine [17]. The degree to which the approach to maternity care reported by CM practitioners is reflected in the experience of woman receiving the care, however, remains unexamined although preliminary research reports clients visiting with $\mathrm{CM}$ practitioners experience a relationship which fits within the patient-centred care paradigm [10, 18], which has been previously identified as a driver for CM use by clients [20].

A feature of the woman-centred care approach identified in our study was the emphasis the participants placed on informed decision-making. An important characteristic of woman-centred care, informed decision-making is linked with a woman's sense of empowerment and control and overall birth satisfaction [1]. Previous research indicates women who access CM practitioners for pregnancy care value the additional information provided through this encounter $[8,11]$. However, the wider health and research community lack details about the specific knowledge and training of $\mathrm{CM}$ practitioners with regards to maternity care and as such the accuracy and validity of the information being purveyed is unclear and requires further research $[21,28]$. Additionally, current evidence suggests users of $\mathrm{CM}$ tend to have higher levels of education and, as such, greater health literacy than the general population [12]. As such, the practice of providing and supporting informed decision-making within $\mathrm{CM}$ may be of value to the women accessing $\mathrm{CM}$ for their maternity care. Further, the emphasis placed by CM practitioners on educating women in their care may be a 'pull' factor that attracts individuals to access their services $[10,11]$ and may be potentially more compelling if women are not feeling informed and empowered in their decision-making by conventional maternity care providers [29]. 
The experiences of women receiving maternity care from a CM practitioner warrants examination to understand how the self-reported care approach described by the participants in this study is actually experienced by women. Additionally, if women are choosing to engage with $\mathrm{CM}$ practitioners to complement their existing mainstream maternity services, the interprofessional dynamics between $\mathrm{CM}$ practitioners and other maternity health professionals providing care to the same woman also requires researcher attention.

Contemporary maternity health services research highlights the value and importance of continuity of care $[2,4]$, which was described by the participants in our study as a feature of their therapeutic relationship with women in their care. Continuity of maternity care has been found to improve not only women's experience and satisfaction with birth but also to provide better health outcomes including reduced rates of intervention, lower incidence of postnatal depression, and overall lower morbidity [30]. Within maternity care this continuity primarily focuses on the same provider involved in care throughout the pregnancy, birth and early postnatal period [31]. Our study suggests CM practitioners may be extending this continuity to both preconception and later postnatal period. In some instances, the women had been longstanding clients of the CM practitioner and as such their therapeutic relationship may mirror the role of a GP or the primary care practitioner for some individuals [4]. According to our study findings, CM practitioners may also be actively encouraging conversations pertaining to preconception care with the women in their care. Current research suggests this particular aspect of family medicine is largely overlooked or underemphasised in contemporary health care [32] and as such the potential contribution of $\mathrm{CM}$ practitioners in filling this gap warrants closer examination. 
This study is not without limitations. The sample of CM practitioners were only drawn from a bound geographical area and as such cannot be confidently claimed to represent the experiences of practitioners in other areas or countries. By the nature of the qualitative study design, the findings are also not able to be generalised to wider community. This study also provides little insight into the outcomes of maternity care from a CM practitioner, instead focusing on the approach to care, and as such should not be interpreted as evidence for or against the safety and efficacy of CM in this population. Further, this study also only captures the self-reported perspective of CM practitioners and as such should not be interpreted as evidence that their approach to maternity care as described in this study are necessarily reflective of their daily practice or experienced by the women in their care.

\subsection{Conclusion}

CM practitioners' approach to maternity care appears to be congruent with the principles of woman-centred care. Given the value placed on woman-centricity in contemporary maternity care, this alignment warrants further examination. Additional research is also needed which examines the effectiveness and safety of CM to better understand the potential contribution of $\mathrm{CM}$ practitioners to increasingly burdened maternity care systems.

\subsection{Practice Implications}

Health professionals and policy makers interested in quality, woman-centred maternity care may need to broaden their gaze to consider the role of CM practitioners in contemporary maternity care provision. Should future research confirm women's experience of maternity care from a CM practitioner matches the perceptions reported in this study, then closer attention should be paid to the factors which enable CM practitioner to provide this type of care and the barriers to such care being more widely experienced within conventional maternity care services. 
Equally, as women are accessing care from CM practitioners during pregnancy, additional support should be given to the education and regulation of $\mathrm{CM}$ to ensure the clinicians are providing information and treatments that are safe, appropriate and coordinated with the wider maternity care service landscape.

\section{Acknowledgements}

Thank you to the participants who gave their time to be involved this project.

Conflicts of Interest

No authors have a conflict of interest to declare.

Funding

[redacted for blinded review] was supported through an Australian Postgraduate Award scholarship provided by the Australian government while undertaking data collection. We also acknowledge the ARC for funding this project via Discovery Project Funding [redacted for blinded review]. 
Table 1: Participants grouped by profession

\begin{tabular}{|l|c|}
\hline Professional group & Number of \\
& participants \\
\hline Acupuncturist & 6 \\
\hline Doula & 4 \\
\hline Chiropractor & 4 \\
\hline Massage therapist & 3 \\
\hline Naturopath & 4 \\
\hline Osteopath & 2 \\
\hline
\end{tabular}


References

1. Shaw D, Guise J-M, Shah N, Gemzell-Danielsson K, Joseph KS, Levy B, Wong F, Woodd S, Main EK: Drivers of maternity care in high-income countries: can health systems support woman-centred care? Lancet 2016, 388:2282-2295.

2. Renfrew MJ, McFadden A, Bastos MH, Campbell J, Channon AA, Cheung NF, Silva DRAD, Downe S, Kennedy HP, Malata A: Midwifery and quality care: findings from a new evidence-informed framework for maternal and newborn care. The Lancet 2014, 384(9948):1129-1145.

3. Clayman ML, Bylund CL, Chewning B, Makoul G: The impact of patient participation in health decisions within medical encounters: a systematic review. Medical Decision Making 2016, 36(4):427-452.

4. Homer CS: Models of maternity care: evidence for midwifery continuity of care. Medical Journal of Australia 2016, 205(8):370-374.

5. Adams J: Introduction. In: Researching Complementary and Alternative Medicine. edn. Edited by Adams J. Oxon: Routeledge; 2007: xiii-xx.

6. Adams J, Lui C-W, Sibbritt D, Broom A, Wardle J, Homer C, Beck S: Women's Use of Complementary and Alternative Medicine During Pregnancy: A Critical Review of the Literature. Birth 2009, 36(3):237-245.

7. Steel A, Adams J, Sibbritt D, Broom A, Gallois C, Frawley J: Utilisation of complementary and alternative medicine (CAM) practitioners within maternity care provision: results from a nationally representative cohort study of 1,835 pregnant women. In: BMC Pregnancy Childbirth. vol. 12; 2012: 146.

8. Steel A, Frawley J, Sibbritt D, Adams J: A preliminary profile of Australian women accessing doula care: findings from the Australian Longitudinal Study on Women's Health. Aust N Z J Obstet Gynaecol 2013, 53:589-592.

9. Provision of maternity care

[http://www.health.gov.au/internet/publications/publishing.nsf/Content/pacdmaternityservicesplan-toc $\sim$ pacd-maternityservicesplan-chapter3]

10. Foley $\mathrm{H}$, Steel $\mathrm{A}$ : Patient perceptions of clinical care in complementary medicine: A systematic review of the consultation experience. Patient Educ Couns 2016, 100:212-223.

11. Steel A, Adams J, Sibbritt D, Broom A, Gallois C, Frawley J: Determinants of women consulting with a complementary and alternative medicine practitioner for pregnancy-related health conditions. Women Health 2013, 54:127-144.

12. Frawley J, Adams J, Sibbritt D, Steel A, Broom A, Gallois C: Prevalence and determinants of complementary and alternative medicine use during pregnancy: Results from a nationally representative sample of Australian pregnant women. Aust N Z J Obstet Gynaecol 2013, 53:347-352.

13. Ivanová $K$, Olecká I, Vencová $B$, Juríčková L: Health literacy of mothers in the first year of motherhood-Expert interpretation map. Kontakt 2018, 20(4):e333e339. 
14. Guerra-Reyes L, Christie VM, Prabhakar A, Siek KA: Mind the gap: Assessing the disconnect between postpartum health information desired and health information received. Women's Health Issues 2017, 27(2):167-173.

15. Bains SS, Egede LE: Association of health literacy with complementary and alternative medicine use: a cross-sectional study in adult primary care patients. BMC Complement Altern Med 2011, 11:138.

16. Long AF: The potential of complementary and alternative medicine in promoting well-being and critical health literacy: a prospective, observational study of shiatsu. BMC Complement Altern Med 2009, 9(1):19.

17. Foley H, Steel A: The Nexus Between Patient-Centered Care and Complementary Medicine: Allies in the Era of Chronic Disease? J Altern Complement Med 2017, 23:158-163.

18. Foley H, Steel A: Patient perceptions of patient-centred care, empathy and empowerment in complementary medicine clinical practice: A cross-sectional study. Advances in Integrative Medicine 2017, 4:22-30.

19. Braddock III CH, Edwards KA, Hasenberg NM, Laidley TL, Levinson W: Informed decision making in outpatient practice: time to get back to basics. J Amer Med Assoc 1999, 282:2313-2320.

20. Bishop FL, Yardley L, Lewith GT: A systematic review of beliefs involved in the use of complementary and alternative medicine. J Health Psychol 2007, 12:851867.

21. Adams J, Steel A: Investigating complementary and alternative medicine in maternity care: The need for further public health/health services research. Complement Ther Clin Pract 2012, 18:73-74.

22. Pope C, Mays N: Qualitative research in health care: John Wiley \& Sons; 2013.

23. Bick D, Howard LM, Oram S, Zimmerman C: Maternity care for trafficked women: Survivor experiences and clinicians' perspectives in the United Kingdom's National Health Service. PloS one 2017, 12(11):e0187856.

24. Rubashkin N, Torres C, Escuriet R, Dolores Ruiz-Berdún M: "Just a little help": A qualitative inquiry into the persistent use of uterine fundal pressure in the second stage of labor in Spain. Birth 2019, https://doi.org/10.1111/birt.12424.

25. Hunter A, Devane D, Houghton C, Grealish A, Tully A, Smith V: Woman-centred care during pregnancy and birth in Ireland: thematic analysis of women's and clinicians' experiences. BMC pregnancy and childbirth 2017, 17(1):322.

26. Kitson A, Marshall A, Bassett K, Zeitz K: What are the core elements of patientcentred care? A narrative review and synthesis of the literature from health policy, medicine and nursing. J Adv Nurs 2013, 69:4-15.

27. Miller S, Abalos E, Chamillard M, Ciapponi A, Colaci D, Comandé D, Diaz V, Geller S, Hanson C, Langer A et al: Beyond too little, too late and too much, too soon: a pathway towards evidence-based, respectful maternity care worldwide. The Lancet 2016, 388(10056):2176-2192. 
28. Steel A, Adams J: The role of naturopathy in pregnancy, labour and postnatal care: broadening the evidence-base. Complement Ther Clin Pract 2011, 17:189192.

29. Barry MJ, Edgman-Levitan S: Shared decision making - the pinnacle of patientcentered care. N Engl J Med 2012, 366:780-781.

30. Sandall J, Soltani H, Gates S, Shennan A, Devane D: Midwife-led continuity models versus other models of care for childbearing women. Cochrane Libr 2016.

31. Waldenström U, Turnbull D: A systematic review comparing continuity of midwifery care with standard maternity services. BJOG 1998, 105:1160-1170.

32. Steel A, Lucke J, Reid R, Adams J: A systematic review of women's and health professional's attitudes and experience of preconception care service delivery. Fam Pract 2016, 33(6):588-595. 